\title{
High-Grade Spondylolisthesis in Adults: Current Concepts in Evaluation and Management
}

\author{
KYLE N. KUNZE, MD, ${ }^{1}$ DANIEL T. LILLY, MD, ${ }^{2}$ JANNAT M. KHAN, MD ${ }^{3}$ PHILIP K. LOUIE, MD, ${ }^{1}$ \\ JOSEPH FERGUSON, MD,${ }^{4}$ BRYCE A. BASQUES, MD,${ }^{3}$ MICHAEL T. NOLTE, MD,${ }^{3}$ \\ CHRISTOPHER J. DEWALD, MD ${ }^{3}$ \\ ${ }^{1}$ Department of Orthopaedic Surgery, Hospital for Special Surgery, New York, New York. ${ }^{2}$ Department of Neurosurgery, Cleveland Clinic Foundation, Cleveland, \\ Ohio. ${ }^{3}$ Department of Orthopaedic Surgery, Rush University Medical Center, Chicago, Illinois ${ }^{4}$ MedStar Georgetown University Hospital, Washington, \\ District of Columbia
}

\begin{abstract}
Background: Information regarding the treatment of high-grade spondylolisthesis (HGS) in adults has been previously described; however, previous descriptions of the evaluation and surgical management of HGS do not represent more recent and now established approaches. The purpose of the current review is to discuss current concepts in the evaluation and management of patients with HGS.

Methods: Literature review.

Results: HGS is diagnosed in up to $11.3 \%$ of adults with spondylolisthesis and typically presents as nonspecific lower back pain. Regarding evaluation, a thorough history and physical examination should be performed, which may help predict the presence of HGS. Diagnostic imaging, and specifically the use of spino-pelvic parameters, are now commonly implicated in guiding treatment course and prognosis. When surgical intervention is indicated, surgical approaches include in situ fusion variations, reduction and partial reduction with fusion, and vertebrectomy. Although the majority of studies suggest improvements with these approaches, the literature is limited by a low level of evidence with regards to the superiority of one technique when compared with others.

Conclusions: HGS is a unique cause of low back pain in adults that carries considerable morbidity, but rarely presents with neurologic symptoms. Although the definitions, classifications, and methods of diagnosis of this spinal deformity have been established and accepted, the ideal surgical management of this deformity remains highly debated. Fusion in situ techniques are often technically easier to perform and provide lower risk of neurologic complications, whereas reduction and fusion techniques offer greater restoration of global spino-pelvic balance. Preoperative spinopelvic parameters may have utility in assisting in procedural selection; however, future, higher-quality and longer-term studies are warranted to determine the optimal surgical intervention among the widely available techniques currently used, and to better define the indications for these interventions.
\end{abstract}

Lumbar Spine

Keywords: spondylolisthesis, thoracolumbar, low back pain

\section{INTRODUCTION}

High-grade spondylolisthesis (HGS) is an uncommon cause of lower back pain in adults. Spondylolisthesis refers to the forward translation of one vertebral body relative to another directly below. Most often, spondylolisthesis results secondary to an anatomic defect in the pars interarticularis of the lumbar spine. "The specifier "high-grade" implies a slippage of one vertebral body by greater than $50 \%{ }^{2}$ HGS is found in approximately $11.3 \%$ in those with spondylolisthesis. ${ }^{1}$

Multiple etiologies can lead to HGS. Most often, either congenital anomalies predisposing to spondylosis and slip progression or gradual degeneration of the posterior facet joints (dysplastic), or defects in the pars interarticularis (isthmic) are responsible. However, other types such as traumatic and pathologic also exist. ${ }^{3}$ With the rapid evolution of surgical techniques, in conjunction with developments in nuclear medicine and diagnostic modalities, the evaluation and management of this deformity in adults has become increasingly complex.

The purpose of the current article is to provide a comprehensive review on current concepts in the management and evaluation of the adult patient population with HGS. The review first presents the basic terminology and classification systems used to discuss spondylolisthesis in adults in order to 
provide a clear understanding of the pathology. Furthermore, the review will discuss approaches to the clinical evaluation of patients with HGS using key findings in the history and physical to guide management. A brief overview of the various diagnostic imaging modalities and their use to this end will also be provided. Finally, both nonoperative and operative treatment of HGS will be discussed, with references to recent clinical studies using these techniques. Together, this review will provide a better background and understanding of HGS in adults and an update on currently accepted concepts in evaluation and management.

\section{BASIC TERMINOLOGY AND CLASSIFICATIONS}

The term spondylolisthesis refers to the forward translation of one vertebral body over the one beneath. ${ }^{1}$ This pathoanatomical displacement of a vertebral segment distinguishes this process from spondylolysis, which refers to a defect in the pars interarticularis without displacement of the vertebral body. ${ }^{1}$ When referring to spondylolisthesis, the severity of the defect may be classified as low-grade or high-grade. According to the Meyerding classification, HGS is defined as vertebral translation with greater than $50 \%$ displacement. ${ }^{4,5}$ In particular, this is based off of the percentage of displacement of the inferior aspect of the body of L5 in relation to the superior border of the sacrum and is graded as follows: no displacement is grade $0 ; 0 \%$ to $25 \%$ displacement is grade I; $26 \%$ to $50 \%$ is grade II; $51 \%$ to $75 \%$ is grade III; $76 \%$ to $100 \%$ is grade IV; and more than $100 \%$ is grade V (spondyloptosis). ${ }^{5}$

In terms of etiology, the Wiltse-Newman classification system ${ }^{3}$ is widely accepted when describing the origin of spondylolisthesis in patients. They define 6 etiologies: type I (dysplastic), type II (isthmic), type III (degenerative), type IV (traumatic), type V (pathologic), and type VI (iatrogenic). ${ }^{3}$ Type I HGS refers to HGS that is secondary to congenital anomalies. More specifically, this may be secondary to misoriented or hypoplastic facets, sacral deficits, or a poorly developed pars interarticularis, which allow for elongation or eventual separation and forward slippage of the lumbosacral joint with repetitive loading over time. ${ }^{3}$ Type II HGS refers to HGS secondary to defects in the pars interarticularis. ${ }^{3}$ Type II HGS is further subclassified into type IIA, type IIB, and type IIC. Type IIA is the most common type, resulting from fatigue failure of the pars interarticularis from repetitive loading and resulting in a complete radiolucent defect. ${ }^{3}$ Type IIB is the result of an elongated pars interarticularis secondary to repeated microfractures that heal and can be difficult to distinguish from type I on plain radiographs. ${ }^{3}$ Type IIC is the result of a pars interarticularis fracture from an acute injury, elongation of the pars, or a fatigue fracture of the pars. ${ }^{3}$ Type III is secondary to degeneration of the disc and facets, which may create instability and mobility on segment. Type IV is secondary to an acute fracture (at a location other than the pars, like type IIC), usually involving the pedicles, facets, or blades. Type $\mathrm{V}$ is secondary to pathologic disruptions such as neoplastic and metabolic processes. Type VI is iatrogenic in which disruptions to the musculature, ligaments, facet capsules (removal of $>50 \%$ of facet joints), removal of $>5 \mathrm{~mm}$ of the pars interarticularis, or disruption of the disc result in subsequent destabilization and slippage. ${ }^{3,6}$

The Marchetti and Bartolozzi ${ }^{7}$ classification system combines the dysplastic and isthmic categories of the Wiltse-Newman classification system and further divides spondylolisthesis into 2 etiology types: developmental and acquired. According to this classification, HGS occurs when there is greater than $50 \%$ vertebral displacement in the context of inherited bony dysplasia of the pars, lumbar facets, discs, and vertebral end plates. ${ }^{7}$ The developmental etiology encompasses the vast majority of cases of HGS because it is relatively unusual for the acquired type to progress to the extent of greater than $50 \%$ slippage. This classification defines the acquired etiology as a failure of the pars interarticularis secondary to repetitive spinal loading related to specific activities. ${ }^{7}$ Although both the Marchetti and Bartolozzi and Wiltse-Newman systems are descriptive, neither were designed to assist in surgical planning. ${ }^{8}$

The Spinal Deformity Study Group (SDSG), ${ }^{9}$ a group of 43 spine surgeons, have proposed a classification system in 6 different sagittal postures based on the radiographic measurement of slip grade and spino-pelvic alignment, which was constructed to help guide surgical decision making in L5-S1 spondylolisthesis. This is based on patterns of progression and associated compensatory mechanisms, which may influence the degree to which one may need to reduce the deformity. They classify 
spondylolisthesis as either low grade $(<50 \%)$ or high grade $(>50 \%)$. Under the low-grade distinction, they define 3 types based on the pelvic incidence (PI): type I (PI < 45) (nutcracker), type II $(\mathrm{PI}=45-60)$, and type III $(\mathrm{PI}>60)$. Under the high distinction, they define 3 more types: type IV (balanced pelvis), type $\mathrm{V}$ (retroverted pelvis with balanced column), and type VI (retroverted pelvis with unbalanced column). Bao et $\mathrm{al}^{10}$ conducted a study to assess the intraobserver and interobserver reliability of the SDSG system in a cohort of 80 patients with lumbar spondylolisthesis. The authors found that the SDSG system provided excellent intraobserver and interobserver reliability, especially in classifying L5-S1 spondylolisthesis, and provided significant clinical utility.

\section{CLINICAL EVALUATION}

Adult patients with HGS typically present with complaints of low back pain that may occasionally be accompanied by radicular symptoms. ${ }^{6,7}$ Typically, the severity of pain and symptoms correlates with the degree of vertebral displacement. ${ }^{11}$ However, in some cases of HGS, spino-pelvic balance cannot be maintained by a compensatory lumbar lordosis (LL), pelvic retroversion, or thoracic kyphosis. In such cases, although patients may remain asymptomatic secondary to compensatory changes in the lumbar anatomy over time, degenerative changes with aging may result in the inability to adapt with further changes, resulting in the development of generalized lumbar back pain. ${ }^{7}$ When global sagittal deformity resulting from anatomic changes to lumbosacral or pelvic anatomy secondary to HGS occurs, this is a differentiating factor from lower grades of spondylolisthesis, which rarely present with such deformity. ${ }^{12}$

Even prior to obtaining imaging, certain factors may help the clinician predict the presence of HGS. Elements in the history may include an insidious onset of symptoms, which is most common in the adult population. ${ }^{6}$ A record of specific physical activities and sports participation is also helpful because many patients may have endured past highgrade disc slips that have since stabilized. ${ }^{6}$ Activities requiring hyperextension and persistent lordosis also cause shearing forces to be applied to the neural arch, and these having been implicated as a causative factor in the eventual development of spondylolisthesis in susceptible patients. ${ }^{13}$ It is notable that although some patients experience radicular symptoms, these are rare. In fact, most patients present with nonspecific back pain alone because bowel and bladder symptoms and saddle anesthesia are uncommon presentations and may indicate alternate pathologies. ${ }^{14}$ Furthermore, night pain is atypical, and this is more likely to suggest the presence of occult neoplasm rather than HGS. ${ }^{6}$

Findings in the physical examination may also help to narrow the differential diagnosis. In patients with HGS, the sacrum may appear vertically oriented, and in an advanced slip, a visible or palpable step-off at the spinous processes of the involved levels, for example, with the process of L5 being more prominent than that of L4, may be observed. ${ }^{7}$ After visualization of the lumbar region, palpation of the lumbar spine and surrounding areas may elicit tenderness in the lumbodorsal fascia, the paraspinal muscles, and the sacroiliac joint, which may further suggest a high-grade slip, although this finding is nonspecific. ${ }^{6,7}$ Additionally, lumbar flexion and extension is often significantly limited, and pain exacerbation is elicited with lumbar hyperextension, ${ }^{15}$ especially with the onelegged hyperextension test. ${ }^{7}$ A gait assessment should also be conducted with visualization of the lumbar spine because altered gait biomechanics secondary to global sagittal misalignment is common in HGS. ${ }^{16}$ In an attempt to compensate for such misalignment, patients may adopt a widebased standing posture with marked flexion at the hip and knee joints. ${ }^{7}$ This results in a crouching gait with short stride-length because the decrease in LL and subsequent posterior pelvic tilt (PT) lead to hamstring tightness and knee flexion. ${ }^{16}$

Neurologic symptoms may also present as sequalae of HGS depending on the degree of neural compromise; however, it should be recognized that radicular symptoms or sensory deficits may suggest alternate pathologies and are nonspecific. ${ }^{6}$ When these symptoms do present secondary to HGS, it is important to note that an L5 radiculopathy can indicate either degenerative or isthmic etiologies. In degenerative etiologies, the L5 radiculopathy occurs secondary to central compression whereby L4 slips forward on L5, resulting in the L4-L5 bulging disc, which together with the posterior elements compromises the central canal. ${ }^{7}$ Degenerative spondylolisthesis may also result in retrolisthesis in which the superior vertebrae slides backward on the one below. This may result in central compression as well; however, retrolisthesis more often compresses 
the nonexiting nerve root, in this scenario, S1. In isthmic etiologies, foraminal stenosis ensues, resulting in similar physical examination findings. In these cases, the L5 nerve root is more commonly compressed because of a combination of the cartilaginous tissue within the pars, which allows direct compression of the nerve root between the pedicle or the pars as L5 slips forward on S1. ${ }^{7}$

\section{Diagnostic Imaging}

Plain radiographs are used as the first-line imaging modality in cases of HGS, in which standing (weight-bearing) anterior-posterior (AP), lateral films, and flexion/extension views of the thoracic and lumbar spine should be taken. ${ }^{6}$ In addition, the examiner may opt to request a Ferguson view of the sacrum, supine views, oblique views, and a long cassette standing radiograph of the entire spine. However, standing radiographs are always necessary in order to determine overall sagittal alignment of the spine. ${ }^{6}$

The standing AP film is useful to evaluate for overall coronal alignment and co-existing scoliosis ${ }^{6}$ in the presence of HGS, which may occur secondary to paraspinal muscle spasms as the result of asymmetric forward vertebral body translation. When HGS occurs at the lumbosacral junction, the so-called "inverted Napoleon's hat" sign may be present on an AP film. This is formed by severe subluxation of the L5 vertebral body leading to projection of the anterior cortex over the sacrum. . $^{8,17}$ The Ferguson view, in which the x-ray beam is aimed parallel to the L5-S1 segment providing a $20^{\circ}$ caudocephalic anteroposterior $\mathrm{x}$-ray, may provide additional evidence of spondylolisthesis and the size of the lumbar transverse processes and is useful to assess degree of postoperative fusion. ${ }^{6,8}$ The standing lateral film is useful in the evaluation sagittal alignment, pars defects, PI, and the degree of spondylolytic defects in addition to the degree of spondylolisthesis per the Meyerding classification. ${ }^{4,6}$ Lateral flexion/extension views may be used to assess segment mobility.

The use of plain radiographs and measurement of spine-pelvic parameters preoperatively often guide treatment and predict prognosis. For example, the radiologist or examiner may measure the slip angle on the standing lateral radiograph of the thoracolumbar spine, which quantifies that degree of lumbosacral kyphosis that has occurred in the presence of forward vertebral translation. ${ }^{6}$ Given

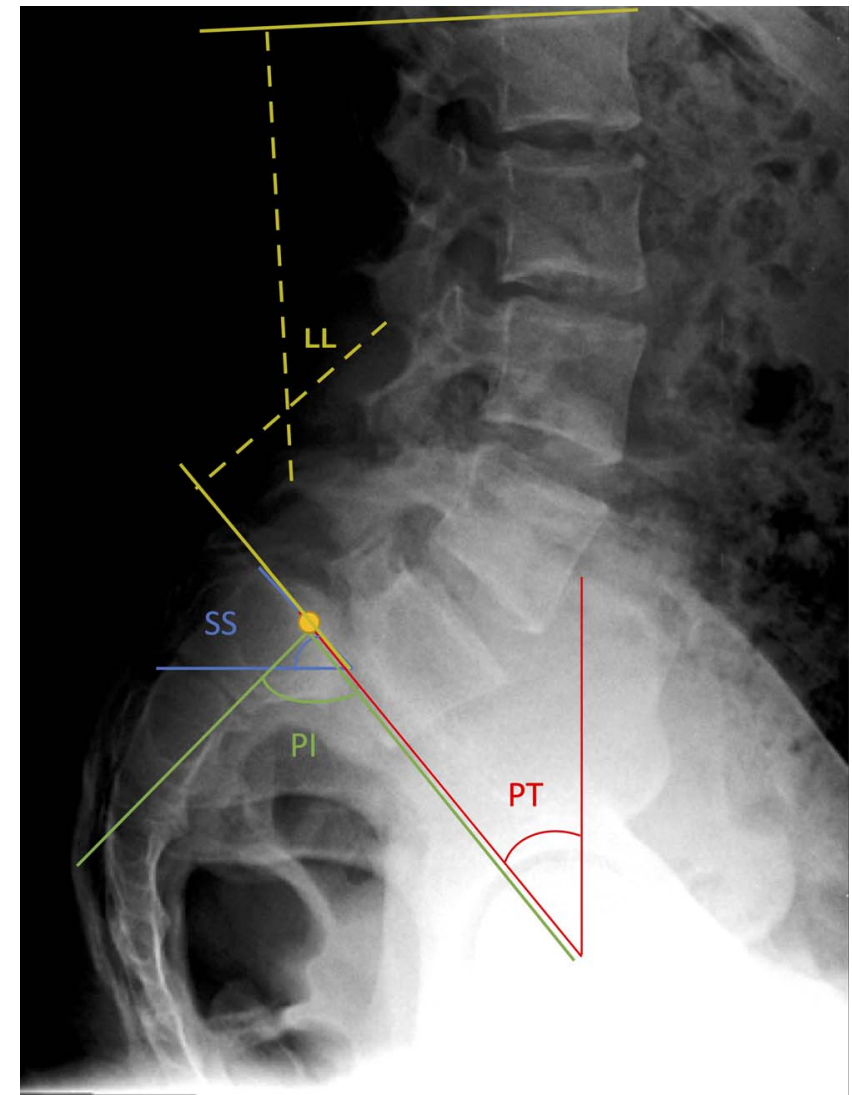

Figure 1. Spino-pelvic radiographic parameters measured on a lateral lumbar radiograph of an individual with high-grade spondylolisthesis (HGS). Lumbar lordosis (LL) is the sagittal Cobb angle measured between the superior end plate of L1 and the superior end plate of S1. The sacral slope (SS) is measured by creating an angle between the center of the sacral plateau and the horizontal. The pelvic tilt (PT) is measured as the angle between the line passing through the center of the femoral heads and the center of the sacrum. The pelvic incidence $(\mathrm{PI})$ is measured as the angle between a line drawn perpendicular to the sacral end plate at its midpoint and a line drawn from the midpoint of the sacral end plate to the midpoint of the femoral head axis.

this information, various spino-pelvic parameters have also become highly implicated in guiding treatment and predicting prognosis for cases of HGS (Figure 1).

On lateral standing radiographs, the LL is the sagittal Cobb angle measured between the superior end plate of $\mathrm{L} 1$ and the superior end plate of $\mathrm{S} 1^{18}$; normal LL values range between $26^{\circ}$ and $76^{\circ}$ with a mean of $46.5^{\circ} .^{19}$ It should be noted that this presents a clinical problem in the case of HGS because the end plate is often curved or irregular and measurements may be inaccurate. The sacral slope (SS) is the angle of the sacral plateau to the horizontal, which is a measurement of the position of the lumbar spine ${ }^{20}$; normal values range from $19.5^{\circ}$ to $65.5^{\circ}$ with a mean value of $39.4^{\circ} .{ }^{19}$ The PT is measured as the angle between the line passing through the center of the femoral heads and the 
center of the sacrum ${ }^{21,22}$; normal values range from $-1^{\circ}$ to $27.9^{\circ}$ with a mean value of $12.3^{\circ}{ }^{19}$ The PI is measured as the angle between a line drawn perpendicular to the sacral end plate at its midpoint and a line drawn from the midpoint of the sacral end plate to the midpoint of the femoral head axis $^{18,21-23}$; normal values range from $33^{\circ}$ to $85^{\circ}$ with a mean value of $51.7^{\circ} .{ }^{19}$ HGS is associated with an abnormally high PI, ${ }^{9}$ with 1 recent cohort study of 276 patients demonstrating that almost all patients have above average PI values of $>60^{\circ} .{ }^{24}$ Indeed, both the postoperative PI and PT have been demonstrated to influence the development of early adjacent segment disease. Together, these findings demonstrate that determining the sagittal alignment through such measurements have important prognostic value and may influence surgical approach to treatment, as outlined by the SDSG system.

It is well known that patients with HGS have global sagittal imbalance through abnormal sacropelvic morphology and loss of the normal anatomic curvature of the spine. ${ }^{25}$ Patients with HGS can be further divided based on whether the pelvic version is "balanced" or "unbalanced." This is determined on the basis of the radiographic parameters of SS and PT, ${ }^{9}$ with the algebraic sum of these 2 angles being the PI. ${ }^{20}$ Patients with balanced HGS have a high SS (mean value of $59.9^{\circ}$ ) and low PT (mean value of $21.3^{\circ}$ ), which has been shown to be similar to the anatomy of healthy control subjects, whereas patients with unbalanced HGS have a retroverted pelvis and vertical sacrum, which correlates with a low SS (mean value of $40.3^{\circ}$ ) and high PT (mean value of $\left.36.5^{\circ}\right) .^{9,25}$ It has been proposed that treatment strategies should be based on the biomechanical strain of the spino-pelvic junction. In patients with unbalanced HGS, it has been suggested that reduction strategies should be considered. ${ }^{9}$ By repositioning the L5 spinal segment over S1 in unbalanced HGS, both the pelvic retroversion and abnormally high LL can be decreased leading to improved pelvic balance. ${ }^{26}$ When SS values are instead high, biomechanical studies have indicated that more aggressive fixation techniques may be needed to achieve adequate construct stiffness. ${ }^{27}$

Computed tomography (CT) imaging may also be used for further diagnostic evaluation following $\mathrm{x}$-ray imaging. Single-photon emission CT (SPECT) is one such imaging modality in the evaluation of HGS and may provide effective detection of spondylolysis as when plain radiographs are incon- clusive. ${ }^{28}$ However, there is a paucity of literature regarding the benefit of using SPECT to diagnose HGS in particular. When using SPECT to evaluate possible cases of spondylolisthesis, one will observe increased osteoblastic activity in areas of stress reactions, and increased radionucleotide uptake may indicate healing potential. ${ }^{29,30}$

Thin-section CT is another type of CT imaging performed with the reverse gantry angle. Thinsection $\mathrm{CT}$ is the best imaging modality for defining the bony anatomy in cases of spondylolisthesis. ${ }^{31}$ An understanding of this anatomy in cases of HGS can be especially important when planning a surgical intervention because the L5 pedicle length varies based on the degree of slip. ${ }^{32}$ Serial imaging using thin-section CT may also demonstrate progressive healing of stress reactions with improvement in the severity of spondylolisthesis. ${ }^{31}$ Finally, magnetic resonance imaging (MRI) is an appropriate imaging modality in cases when a patient with HGS presents with associated neurologic symptoms. ${ }^{6}$ MRI allows for the evaluation of neurologic tissue such as the dural sac and traversing nerve roots, as well as the degree of adjacent disc hydration. ${ }^{33}$ Using this imaging modality allows for differentiation between central or foraminal neurologic compromise as seen with degenerative and isthmic spondylolisthesis, respectively. When ordering an MRI of the lumbar spine in cases of spondylolisthesis, it is important to image the entire sacrum because failure to do so risks missing L5-S1 spondylolisthesis and central dural impingement. ${ }^{33}$

\section{Nonoperative Management}

When patients with confirmed HGS present, it is recommended to begin with trials of physical therapy and exercise except in rare cases where a cauda equina syndrome is encountered. ${ }^{34}$ For those without symptoms, it is hypothesized that improved strength of the paraspinal musculature may reduce the likelihood of deformity advancement, but there is little evidence to support this claim. Generally, physical therapy should consist of strengthening, range of motion advancements, and low impact exercise. Specially, lumbar flexion exercises and core stabilization exercises for the lower abdominal, lumbar muscles, hamstrings, and hip flexors should be emphasized. ${ }^{14}$ Low impact aerobic exercises, such as cycling, elliptical training, swimming, and walking, are also highly recommended. ${ }^{14}$ Weight reduction, despite minimal effect on neurological 
concerns, is suggested for improvement of lower back pain. ${ }^{35,36}$

A thoracic-lumbar spinal orthosis may provide relief and control posture when supervised exercise therapy is not being performed. Chiropractic adjustments can provide short-term relief for patients but are often inadequate for providing long-term symptom relief. ${ }^{36}$ If physical and exercise therapy does not provide adequate pain relief, nerve root injections may be pursued, particularly in patients with unilateral radicular symptoms. Of note, nerve root injections are typically more efficacious than epidural injections because it is rare for patients with HGS to develop central canal stenosis; this degree of spondylolisthesis is more often than not associated with pars lysis.

There is no evidence to support prophylactic fusion for cases of asymptomatic HGS to prevent potential slip progression and symptom development, nor is it indicated on the basis of long-term evaluation of these individuals. Despite lack of evidence and available conservative measures, cases of HGS typically demonstrate less reliable improvement than low-grade cases, with $<10 \%$ of nonsurgical cases resulting in symptomatic relief. ${ }^{6}$ Although pain and slip grade is important, sacropelvic morphology and balance are also strong determinants of sagittal spino-pelvic alignment and they should be considered in any treatment algorithm, especially with trials of conservative management. ${ }^{37}$ The specific pattern of sagittal sacropelvic balance for each patient should influence the risk of progression and treatment outcomes. ${ }^{37}$ With a high likelihood of failure of conservative management, and knowledge of spino-pelvic and sagittal parameters indicative of high progression risk, surgical management is often pursued.

\section{Surgical Management}

Indications for surgical management of HGS include persistent symptoms, failure of conservative management, radiographic parameters suggestive of imminent slip progression, significant biomechanical stress, and global sagittal imbalance. This section provides an overview of various surgical techniques used in the treatment of HGS in adults, in particular for progression of isthmic slips, which usually present symptomatically when unbalanced. The most common surgical techniques include (1) in situ fusion techniques using posterolateral, anterior, or circumferential approaches; (2) fusion and

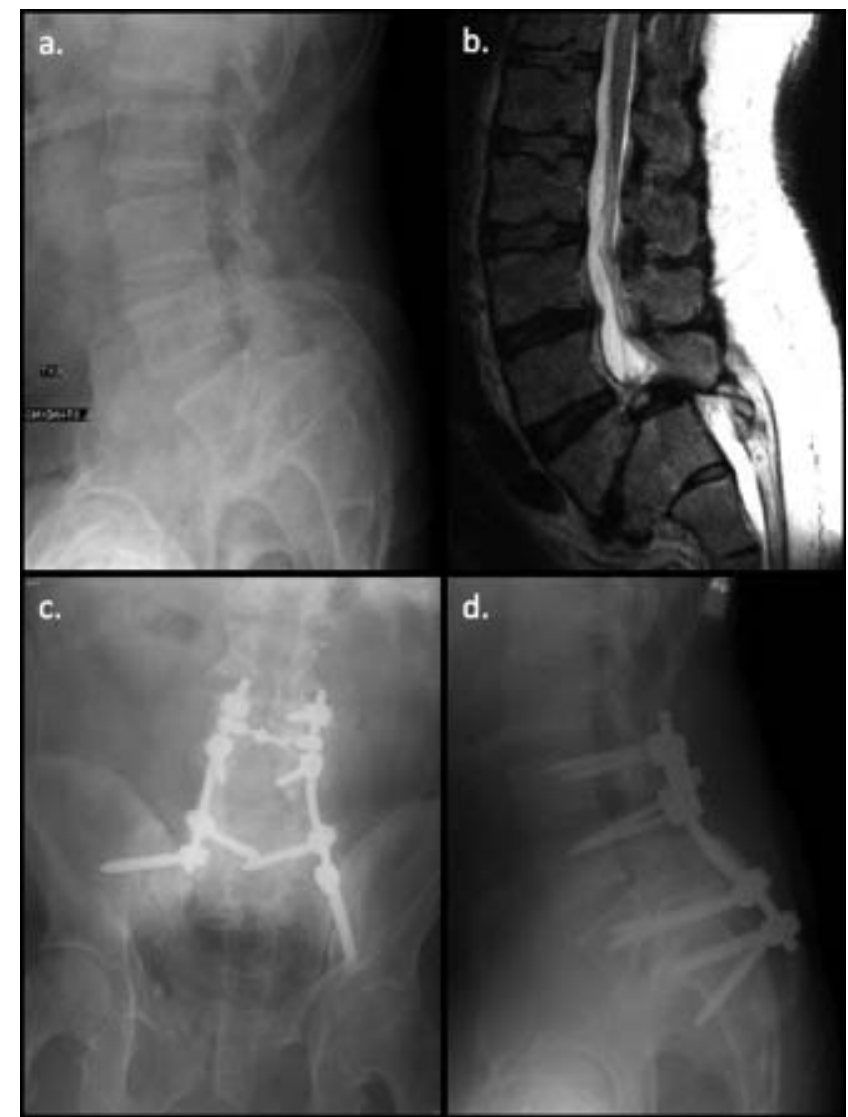

Figure 2. This is a 56-year-old male who presented with right leg weakness and urinary incontinence. (a) Lateral lumbar plain radiograph and (b) T2weighted magnetic resonance imaging of the lumbar spine demonstrated a Meyerding Grade IV spondylolisthesis at L5-S1. As depicted in the (c) anteroposterior and (d) lateral lumbar plain radiographs, he subsequently underwent a laminectomy at L4-S2, a posterolateral fusion from L3-Pelvis, a sacral dome osteotomy, with an additional L5-S1 discectomy and mixed allograft and local autograft placement. No formal reduction maneuver was attempted intraoperatively.

reduction combination techniques; and (3) vertebrectomy.

\section{In Situ Fusion}

The in situ L4-S1 fusion with or without postoperative bracing is a safe and effective surgical intervention for alleviating both the persistent back pain and neurologic symptoms attributable to HGS in adults (Figure 2a-d). Joelson et $\mathrm{al}^{38}$ recently analyzed 35 consecutive patients who underwent in situ arthrodesis for high-grade isthmic spondylolisthesis at a mean follow-up of 29 years (range 23-35 years). At final follow-up, these patients had selfreported outcome scores (Short Form-36 and EuroQol-5 Dimension) that were statistically similar to the age-matched general population, and were equally likely to return to work. In a separate paper, the same group reported on the 30-year radiographic follow-up data for 39 patients who 
underwent in situ arthrodesis for HGS. The authors found that only 3 patients had global sagittal imbalance ( $\mathrm{T} 1$ spino-pelvic inclination $>0^{\circ}$ ) at final follow-up, and that there was a significant decrease in SS at latest follow-up when compared with 8-year follow-up data, ${ }^{39}$ suggesting that this surgical technique is also efficacious in the long-term. A small case series of 6 patients with HGS demonstrated that in situ posterolateral and fibular interbody fusion was an efficacious treatment option for HGS because all patients had solid bony fusion, were asymptomatic, and had no slip progression at a minimum of 1-year follow-up. ${ }^{40}$

Noorian et $\mathrm{al}^{41}$ conducted a recent systematic review of clinical outcomes in the surgical treatment of adult isthmic spondylolisthesis, which included a total of 1538 patients from 6 randomized controlled trials and 9 observational studies. The authors concluded that the available studies provided strong evidence that the addition of reduction to fusion did not result in better clinical outcomes for pain and function. Furthermore, the authors reported that the evidence supported no difference between circumferential fusion and interbody fusion in pain or function but that circumferential fusion was associated with greater intraoperative blood loss and longer hospital stays.

Traditionally, the Wiltse ${ }^{42}$ approach has been the gold-standard for the surgical management of HGS. Generally, in this approach the spinal exposure is made through 2 paravertebral splitting incisions. Unless there are neurological issues, the lamina, pars, and facet joints are left unexposed and bone graft is placed posterior to the transverse processes extending to the sacral alae. Furthermore, placement differs depending on the levels of fusion: L5-S1 in the transverse process fusion, and L4-L5 in facet fusions for L5-S1 HGS. This creates a posterolateral fusion mass that should counteract the shear forces applied at the lumbosacral junction. ${ }^{42}$ More horizontal placement of bone graft should be performed in large slips. This may require extension to L4 in order to have a more vertical graft that is under compression and therefore heals better. It is important to note that exposure of the lamina leads to instability after surgery and is not done unless instrumentation is planned. This approach has the benefits of avoiding destabilization of the posterior elements, and the avascular nature of the paravertebral muscle-splitting approach helps maintain position of the bone graft and may promote fusion.
Rare complications of this surgical procedure include iatrogenic neurologic complications, posterior progression of slippage, pseudarthrosis, and deterioration of the fusion mass. ${ }^{43} \mathrm{~A}$ wide decompression of the nerve roots combined with in situ fusion is another surgical treatment option for patients with HGS and neurologic symptoms, with the benefits of instrumented reduction but a diminished risk of neurologic complications. ${ }^{44}$

\section{Anterolateral Interbody Fusion}

Anterolateral interbody fusion (ALIF) with posterior fixation ${ }^{45}$ is one fusion method used for low dysplasia in the setting on low-grade spondylolisthesis or HGS. This technique uses an abdominal retroperitoneal approach to access the disc space. After the anterior longitudinal ligament, disc, and annulus are removed, and the end plates are prepared for fusion, interbody cages with autograft material are inserted into the affected level. After completion of the ALIF, the patient is turned into the prone position and percutaneous pedicle screws are used to complete a posterior fixation. This method has been shown to provide excellent results in the long-term, with $83 \%$ union rates at 10 -year follow-up. ${ }^{46}$ Riouallon et $\mathrm{al}^{47}$ performed a case series of 65 patients with isthmic spondylolisthesis who underwent ALIF with a mean follow-up of 6.6 years. The authors found that the fusion was $91 \%$, slippage decreased by $30 \%$, disc height increased by $177 \%$, and lordosis have improved by $5^{\circ}$ without changes in pelvic parameters. Furthermore, they noted that the Beaujon Hospital disability index, which is a measure of current functional status scored between 0 and 20 points, ${ }^{48}$ had improved by an average of 7.3 points.

\section{Circumferential Interbody Fusion}

Another variant of in situ fusion techniques is the in situ circumferential interbody fusion. Indications for this procedure include adults with HGS who present with severe back pain and neurologic symptoms. Although multiple circumferential fusion techniques are available, the Bohlman fibular strut remains a popular option. ${ }^{49,50}$ In this technique, a wide decompression of L5-S2 laminectomies is performed in addition to wide L5 foraminotomies. A bony tunnel is then created from the posterior body of S1 to the anterior body of L5, at which point a fibular strut is tamped into the tunnel. The fibular strut provides the benefits of anterior column support to 


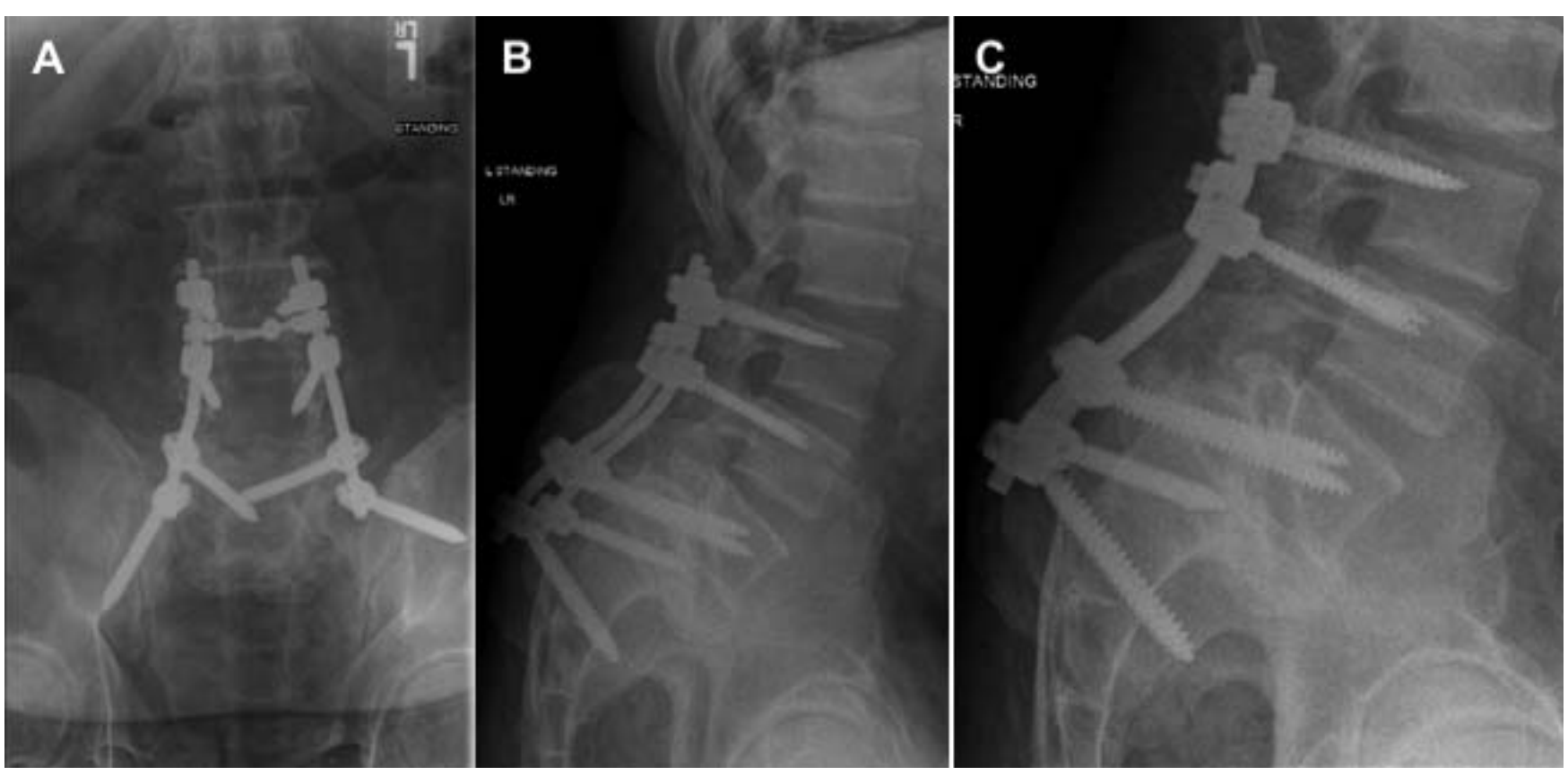

Figure 3. Three views of circumferential interbody fusion using transsacral fixation in a patient with high-grade spondylolisthesis (HGS). (A) Anteroposterior (AP) radiograph of sacral pedicle - vertebral body (transsacral) fixation. (B) Lateral radiograph of the sacral pedicle - vertebral body fixation. (C) Dedicated lumbar radiograph of the transsacral lumbar fusion from L3-S1.

balance the tensile forces that the posterior fusion mass is ultimately exposed to in patients with highgrade slips. Finally, a circumferential fusion is completed by placing posterolateral bone graft from L4 to the sacral alae. This technique also has the advantages of providing a wider surface area for bony fusion, restoring disc height to indirectly decompress neural foramen, and provides anterior pivot to allow a more effective correction of the lumbosacral kyphosis with the use of posterior instrumentation. ${ }^{7}$

The Bohlman technique can be altered or augmented in several fashions. Transsacral S1 or standard pedicle screws can be added to the posterior interbody fusion (Figure 3), which has been shown to improve slip percent and slip angle. ${ }^{51}$ It is also possible to substitute bilateral threaded pedicle screws instead of using a fibula allograft. ${ }^{52}$ Other methods to achieve circumferential fusion include pedicular transvertebral screw fixation, ${ }^{53}$ using a transsacral interbody cage, ${ }^{54}$ or by direct pediculo-body fixation as described by Grob et al ${ }^{55}$ This latter approach provides significantly stiffer fixation than pedicle screws allow, ${ }^{56}$ and the longterm results have been demonstrated to be favorable and reliable. ${ }^{57}$ Higher mechanical stability and faster symptomatic resolution can be achieved by modifying the Grob technique to involve a trans- discal L5-S1 fixation with L5-S1 pedicle screw instrumentation. ${ }^{58}$

To evaluate the efficacy of circumferential fusion, Ferrero et $\mathrm{al}^{59}$ examined the radiological and functional outcomes of HGS in 20 patients treated by intrasacral fixation, dome resection, and circumferential fusion. At a mean follow-up of 7.2 years, the authors found that lumbosacral angle kyphosis and olisthesis were reduced $\left(65^{\circ} \pm 14\right.$ versus $99^{\circ} \pm$ $11, P<.001$ and $81 \% \pm 19$ versus $45 \% \pm 18, P<$ .001 , respectively). The authors also found that 10 patients presented with transient L5 motor deficits, but that all recovered before 3 months postoperatively. They concluded that this circumferential fusion technique was effective in restoring lumbosacral angle kyphosis and global sagittal alignment, as well as resolving compensatory hyperlordosis.

\section{Fusion With Reduction}

More recently, it has been proposed that reduction techniques should be preferably used in individuals with evidence of abnormal posture in order to restore global spino-pelvic balance and improve the biomechanical environment for fusion. ${ }^{37}$ For example, it has been suggested by the SDSG that in patients with increased $\mathrm{LL}$, forceful attempts at reduction may not be required and simple instrumentation after postural reduction may be sufficient to maintain adequate sagittal alignment. ${ }^{37}$ However, 
once the maximum degree of LL is attained, the patient may then attempt to maintain balanced posture through progressive pelvic retroversion. In these cases, posture, reduction, and realignment procedures should preferably be attempted; however, in difficult cases, instrumentation and fusion after postural reduction may also be sufficient to achieve adequate sagittal alignment because spinal alignment is maintained. The indications for instrumented reduction and fusion techniques are as follows: (1) high slip angle $>45^{\circ}$ or severe sagittal imbalance; (2) increased risk for pseudarthrosis with in situ fusion (preoperative L5 radiculopathy or sacral root symptoms requiring wide decompression, high-grade dysplastic spondylolisthesis, hypermobility of L5-S1 segment, and anatomic factors such as small transverse processes, sacral dysplasia, trapezoidal L5 vertebral body, and rounding of the sacrum). ${ }^{60}$

Labelle et $\mathrm{al}^{26}$ studied 73 patients with low-grade spondylolisthesis and HGS managed with partial reduction and fusion with instrumentation or casting. The authors found that grade, lumbosacral angle, LL, and SS improved significantly following surgery, suggesting that spino-pelvic balance should be determined in those with HGS and that those with an unbalanced pelvis may benefit from partial reduction. ${ }^{26}$ Finally, when the limit of LL and pelvic retroversion is achieved, the patient may develop sagittal trunk imbalance. In these cases, reduction and realignment procedures are mandatory, where sagittal alignment is severely deformed. ${ }^{37}$

Interestingly, a recent meta-analysis of 3 randomized controlled trials and 9 comparative observational studies comparing the clinical and radiographic outcomes and complication rates between reduction and fusion in situ techniques for spondylolisthesis found no difference in satisfaction or neurologic complication rates, but found significantly improved fusion rates and a decreased postoperative slip angle in the reduction group. ${ }^{61}$ Fan et $\mathrm{al}^{62}$ conducted a cohort study comparing the clinical outcomes of reduction $(n=24)$ versus fusion in situ with minimally invasive transforaminal interbody fusion $(\mathrm{n}=21)$ for isthmic spondylolisthesis. At a mean ( \pm standard deviation) $34.75 \pm$ 8.06 and $31.05 \pm 6.52$-month follow-up in the reduction and fusion groups, respectively, they found no significant differences in patient-reported outcomes, fusion rates, or complication rates, suggesting that reduction may not be a requirement when using in situ fusion for isthmic spondylolisthesis. ${ }^{62}$

Longo et $\mathrm{al}^{63}$ conducted a systematic review to compare fusion in situ with reduction and fusion for HGS. Using 8 eligible studies, they found that fusion with reduction was associated with a significantly greater decrease in slippage percentage and slip angle compared with fusion in situ. Furthermore, the authors found that pseudarthrosis was more frequent in the fusion in situ group when compared with the reduction with fusion group (17.8\% versus $5.5 \% ; P=.004)$.

Martiniani et al ${ }^{64}$ conducted a small cohort study of 16 patients with high-grade, high-dysplasia spondylolisthesis to assess the influence of fusion in situ versus reduction and fusion on spino-pelvic parameters. At a minimum of 2 years' follow-up, the authors found no statistically significant changes in PT, SS, and grade in the fusion in situ group, whereas the reduction with fusion group had significantly decreased PT and increased SS and grade. The authors concluded that balanced deformities should not have reduction, whereas unbalanced deformities could benefit from corrections via reduction.

The instrumented reduction and fusion technique is an approach to correcting HGS with the goal of fusion plus sagittal correction and improved cosmesis as compared with the in situ approaches. ${ }^{60}$ Other benefits of this approach include restoration of normal lumbosacral alignment, which diminishes the shear forces across the fusion mass as compared with in situ approaches. Furthermore, addition of spinal instrumentation allows for more rapid mobilization and rehab, and helps to facilitate and maintain reduction. ${ }^{60}$ In a recent retrospective multicenter review of 25 adults who underwent reduction and fusion for HGS with a mean month follow-up of 21.3 months, the authors found statistically significant improvements in the Meyerding grade, slip angle, and mean Oswestry Disability Index scores associated with surgery. Additionally, there were no revision surgeries required and only 2 complications in total ( 1 intraoperative neurologic deficit and 1 intraoperative vertebral body fracture). ${ }^{65}$

A downside of this procedure is its technical demands. Many approaches have been described, such as preoperative halo-femoral traction with pelvic suspension, next followed by AP fusion during surgical intervention, and placement of a 
brace in hyperextension postoperatively. ${ }^{26}$ Other options include the anterior release with partial reduction and anterior interbody fusion ${ }^{66}$ or a posterior instrumented reduction and fusion without neural element decompression. ${ }^{67}$ Because there is a broad range of methods for reduction, choice is often dependent on surgeon familiarity or preference rather than specific indications for the different possible approaches.

In addition to its increased technical complexity, this procedure typically requires longer surgical times compared with in situ fusion, increased blood loss compared with in situ fusion, and poses the risk for iatrogenic neurologic injury, which correlates with the degree of reduction obtained. ${ }^{68}$ In particular, the most common complication is an isolated L5 radiculopathy, because stretch and subsequent strain of up to $75 \%$ of the nerve may occur during the second half of the reduction. ${ }^{69,70}$ One recent retrospective review of 17 patients who underwent reduction and instrumented transforaminal lumbar interbody fusion of high-grade L5-S1 isthmic spondylolisthesis identified the incidence of L5 radiculopathy with motor deficit to be $29 \%{ }^{71}$ As such, the benefits of reduction must be considered in the context of increased complication risk when compared with in situ fusion, and a recent systematic review on reduction versus in situ fusion for high-grade slips found no significant difference in clinical outcomes between the 2 procedures. $^{72}$

\section{Partial Reduction and Fusion}

The partial reduction and fusion is an adaptation of the reduction and fusion procedure with advantages that include increased fusion rates in patients with an unbalanced pelvis and lower risks of complications compared with a full reduction (Figure 4). ${ }^{73,74}$ In this technique, the patient is positioned prone and intraoperative monitoring such as somatosensory evoked potentials or electromyography is used throughout the procedure. The L4-S2 segments are exposed, and a complete L5 laminectomy, and wide decompression of the bilateral L5 and S1 nerve roots is performed. Subsequently, a L5-S1 discectomy and partial excision of the sacral dome is completed to shorten the L5-S1 segment. Pedicle screws are then placed at L4, L5, and S1. A gradual reduction with cantilever flexion of the sacral-pelvis unit follows via a combination of patient positioning and gentle downward force on the sacrum. This

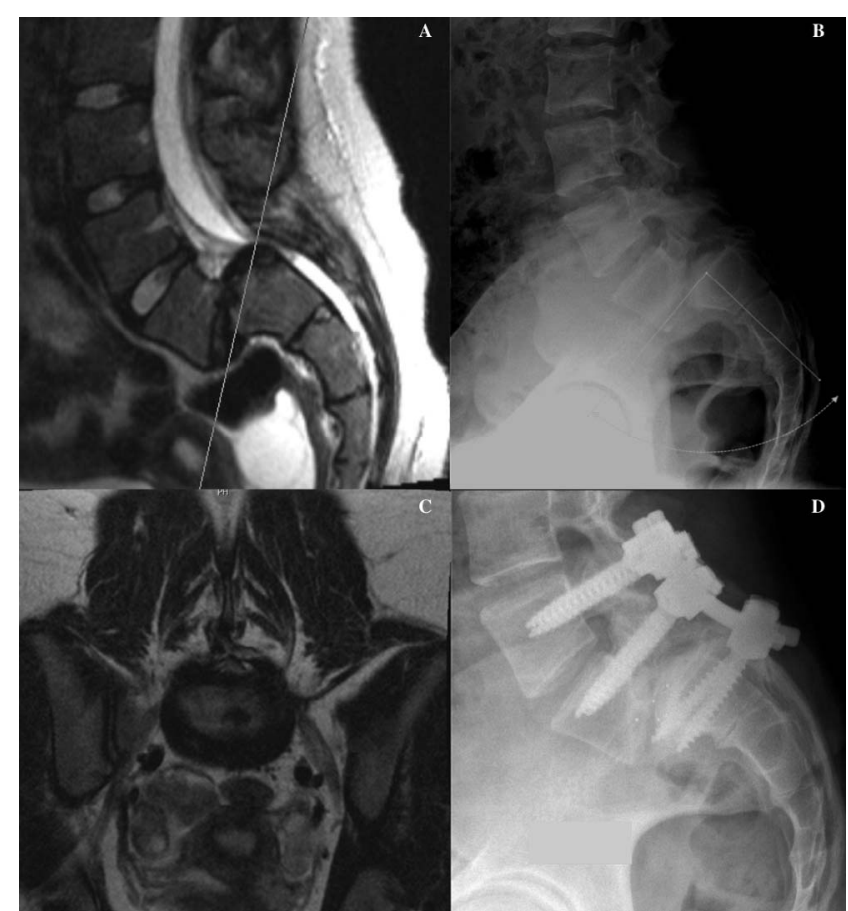

Figure 4. This is a 14-year-old gymnast presented with chronic lower back pain without radicular symptoms. The (A) T2-weighted lumbar magnetic resonance (MR) image and $(B)$ lateral neutral lumbar plain radiograph show exaggerated lordosis and a grade III spondylolisthesis in addition to mild posterior wedging of L5 and mild deformity of the superior S1 end plate. (C) The axial T2-weighted MR image at L5-S1 depicts severe deformity at this level. (D) The patient underwent a laminectomy at L4-S1 with a posterolateral fusion at L4-5 and transforaminal lumbar interbody fusion (TLIF) at L5-S1 following a reduction attempt at L5-S1.

portion of the procedure is performed slowly over 1 to 3 hours intraoperatively to take advantage of soft-tissue relaxation and to allow for neurologic structures to slowly accommodate to the new spinal position. Because nerve root strain is highest at the end of the reduction, a safe surgical goal is typically improvement of the HGS to a grade II displacement (between $25 \%$ and $50 \%$ ), rather than a complete anatomic reduction. After reduction, a bone graft is placed posterolaterally extending from the L4 segment to the sacrum. Finally, a circumferential fusion via posterolateral or transforaminal lumbar interbody fusion, either at the time of initial surgery, or in a second stage by anterior interbody fusion via retroperitoneal approach 7 to 10 days postoperatively, is performed. ${ }^{60}$

Smith et $\mathrm{al}^{74}$ performed a retrospective study of 9 consecutive patients who underwent partial reduction followed by posterior interbody fusion for the treatment of HGS. At a mean follow-up of 43 months (range, 24-72), the authors found that the slip angle improved from $41.2^{\circ}$ to $21^{\circ}$ preoperatively to postoperatively, and that all patients were 
extremely or somewhat satisfied with their surgery. They concluded that partial reduction with fusion is an effective technique for the management of HGS. Another retrospective cases series of 6 patients with HGS who underwent partial reduction and fusion ${ }^{75}$ showed similar results at a mean 42.6 months with significant improvements in slip angle $\left(62^{\circ}\right.$ to $28^{\circ}, P$ $<.05)$ and pain reduction. Furthermore, there was no slip progression or neurologic complications at latest follow-up, but 2 intraoperative dural tears were noted and repaired. ${ }^{75}$

\section{Vertebrectomy}

In severe high-grade slips or cases of spondyloptosis, the Gaines vertebral resection ${ }^{76}$ remains a viable option for correction. The Gaines vertebral resection consists of an anterior retroperitoneal approach with a complete L5 corpectomy and subsequent staged posterior procedure to resect the posterior elements of L5. Because the L5 vertebrae is not in bony contact with the superior end plate of the sacrum in patients with this severe deformity, the posterior end plate of the L4 vertebral body is then placed directly on the superior end plate of the sacrum, and instrumented with pedicle screws into the sacrum. ${ }^{77}$ This procedure has been noted to produce good clinical outcomes and fusion rates, but carries a high incidence of neurological complications $(25 \%)$ following surgery. ${ }^{76}$ Despite this finding, Lehmer et $\mathrm{al}^{78}$ found that patients preferred this deformity correcting procedure over a deformity-preserving procedure, and posited this to be due to restoration of sagittal alignment. A recent case series of 7 consecutive patients with L5-S1 spondyloptosis of Meyerding IV spondylolisthesis treated by a 1-stage L5 spondylectomy demonstrated at mean 65 months follow-up that slip percentage improved from $115 \%$ to $63 \%$ postoperatively and that the PT, LL, and thoracic kyphosis also improved. $^{79}$

\section{CONCLUSION}

HGS is a unique cause of low back pain in adults that carries considerable morbidity, but rarely presents with neurologic symptoms. Although the definitions, classifications, and methods of diagnosis of this spinal deformity have been established and accepted, the ideal surgical management of this deformity remains highly debated. Fusion in situ techniques are often technically easier to perform and provide lower risk of neurologic complications, whereas reduction and fusion techniques offer greater restoration of global spino-pelvic balance. Preoperative spino-pelvic parameters may have utility in assisting in procedural selection; however, future, higher-quality and longer-term studies are warranted to determine the optimal surgical intervention among the widely available techniques currently used, and to better define the indications for these interventions.

\section{REFERENCES}

1. Kalichman L, Kim DH, Li L, Guermazi A, Berkin V, Hunter DJ. Spondylolysis and spondylolisthesis: prevalence and association with low back pain in the adult community-based population. Spine (Phila Pa 1976). 2009;34(2):199-205.

2. Tebet MA. Current concepts on the sagittal balance and classification of spondylolysis and spondylolisthesis. Rev Bras Ortop. 2014;49(1):3-12.

3. Wiltse LL, Newman PH, Macnab I. Classification of spondylolisis and spondylolisthesis. Clin Orthop Relat Res. 1976(117):23-29.

4. Meyerding HW. Spondylolisthesis; surgical fusion of lumbosacral portion of spinal column and interarticular facets; use of autogenous bone grafts for relief of disabling backache. $J$ Int Coll Surg. 1956;26(5 pt 1):566-591.

5. Meyerding HW. Spondylolisthesis. Surg Gynecol Obstet. 1932;54:371-377.

6. DeWald CJ, Vartabedian JE, Rodts MF, Hammerberg $\mathrm{KW}$. Evaluation and management of high-grade spondylolisthesis in adults. Spine (Phila Pa 1976). 2005;30(suppl 6):S49 S59.

7. Marchetti PG, Bartolozzi, P. Classification of Spondylolisthesis as a Guideline for Treatment. 2nd ed. Philadelphia, PA: Lippincott-Raven; 1997.

8. Li Y, Hresko MT. Radiographic analysis of spondylolisthesis and sagittal spinopelvic deformity. J Am Acad Orthop Surg. 2012;20(4):194-205.

9. Hresko MT, Labelle H, Roussouly P, Berthonnaud E. Classification of high-grade spondylolistheses based on pelvic version and spine balance: possible rationale for reduction. Spine (Phila Pa 1976). 2007;32(20):2208-2213.

10. Bao H, Yan P, Zhu W, et al. Validation and Reliability Analysis of the Spinal Deformity Study Group Classification for L5-S1 Lumbar Spondylolisthesis. Spine (Phila Pa 1976). 2015;40(21):E1150-E1154.

11. Harris IE, Weinstein SL. Long-term follow-up of patients with grade-III and IV spondylolisthesis. Treatment with and without posterior fusion. J Bone Joint Surg Am. 1987;69(7):960-969.

12. Gandhoke GS, Kasliwal MK, Smith JS, et al. A multicenter evaluation of clinical and radiographic outcomes following high-grade spondylolisthesis reduction and fusion. Clin Spine Surg. 2017;30(4):E363-E369.

13. Kasliwal MK, Smith JS, Kanter A, et al. Management of high-grade spondylolisthesis. Neurosurg Clin $N \mathrm{Am}$. 2013;24(2):275-291.

14. Metzger R, Chaney S. Spondylolysis and spondylolis- 
thesis: what the primary care provider should know. $J$ Am Assoc Nurse Pract. 2014;26(1):5-12.

15. Miao J, Wang S, Wan Z, et al. Motion characteristics of the vertebral segments with lumbar degenerative spondylolisthesis in elderly patients. Eur Spine J. 2013;22(2):425-431.

16. Beck AW, Simpson AK. High-grade lumbar spondylolisthesis. Neurosurg Clin N Am. 2019;30(3):291-298.

17. Pendergrast TE, Dyer RB. The “inverted Napoleon's hat” sign. Abdom Radiol (NY). 2019;44(8):2931-2932.

18. Schwab F, Patel A, Ungar B, Farcy JP, Lafage V. Adult spinal deformity-postoperative standing imbalance: how much can you tolerate? An overview of key parameters in assessing alignment and planning corrective surgery. Spine (Phila $\mathrm{Pa}$ 1976). 2010;35(25):2224-2231.

19. Vaz G, Roussouly P, Berthonnaud E, Dimnet J. Sagittal morphology and equilibrium of pelvis and spine. Eur Spine $J$. 2002;11(1):80-87.

20. Le Huec JC, Aunoble S, Philippe L, Nicolas P. Pelvic parameters: origin and significance. Eur Spine J. 2011;20(suppl 5):564-571.

21. Jackson RP, Phipps T, Hales C, Surber J. Pelvic lordosis and alignment in spondylolisthesis. Spine (Phila Pa 1976). 2003;28(2):151-160.

22. Jackson RP, Kanemura T, Kawakami N, Hales C. Lumbopelvic lordosis and pelvic balance on repeated standing lateral radiographs of adult volunteers and untreated patients with constant low back pain. Spine (Phila Pa 1976). 2000;25(5):575-586.

23. Aoki Y, Nakajima A, Takahashi H, et al. Influence of pelvic incidence-lumbar lordosis mismatch on surgical outcomes of short-segment transforaminal lumbar interbody fusion. BMC Musculoskelet Disord. 2015;16:213.

24. Labelle H, Roussouly $\mathrm{P}$, Berthonnaud E, et al. Spondylolisthesis classification based on spino-pelvic alignment. Presented at the 2009 Scoliosis Research Society Annual Meeting, San Antonio, TX, USA.

25. Labelle H, Mac-Thiong JM, Roussouly P. Spino-pelvic sagittal balance of spondylolisthesis: a review and classification. Eur Spine J. 2011;20(suppl 5):641-646.

26. Labelle H, Roussouly P, Chopin D, Berthonnaud E, Hresko T, O'Brien M. Spino-pelvic alignment after surgical correction for developmental spondylolisthesis. Eur Spine J. 2008;17(9):1170-1176.

27. Drazin D, Hussain M, Harris J, et al. The role of sacral slope in lumbosacral fusion: a biomechanical study. $J$ Neurosurg Spine. 2015;23(6):754-762.

28. Lusins JO, Elting JJ, Cicoria AD, Goldsmith SJ. SPECT evaluation of lumbar spondylolysis and spondylolisthesis. Spine (Phila Pa 1976). 1994;19(5):608-612.

29. Lowe J, Schachner E, Hirschberg E, Shapiro Y, Libson E. Significance of bone scintigraphy in symptomatic spondylolysis. Spine (Phila Pa 1976). 1984;9(6):653-655.

30. Bodner RJ, Heyman S, Drummond DS, Gregg JR. The use of single photon emission computed tomography (SPECT) in the diagnosis of low-back pain in young patients. Spine (Phila Pa 1976). 1988;13(10):1155-1160.

31. Anderson K, Sarwark JF, Conway JJ, Logue ES, Schafer MF. Quantitative assessment with SPECT imaging of stress injuries of the pars interarticularis and response to bracing. $J$ Pediatr Orthop. 2000;20(1):28-33.

32. Matthews PG, Phan K, Rao PJ, Ball JR. Pedicle length and degree of slip in lumbosacral isthmic spondylolisthesis. Orthop Surg. 2015;7(2):108-111.

33. DeWald RL, Faut MM, Taddonio RF, Neuwirth MG. Severe lumbosacral spondylolisthesis in adolescents and children. Reduction and staged circumferential fusion. J Bone Joint Surg Am. 1981;63(4):619-626.

34. Sairyo K, Katoh S, Takata Y, et al. MRI signal changes of the pedicle as an indicator for early diagnosis of spondylolysis in children and adolescents: a clinical and biomechanical study. Spine (Phila Pa 1976). 2006;31(2):206-211.

35. Agabegi SS, Fischgrund JS. Contemporary management of isthmic spondylolisthesis: pediatric and adult. Spine $J$. 2010;10(6):530-543.

36. Kalichman L, Hunter DJ. Diagnosis and conservative management of degenerative lumbar spondylolisthesis. Eur Spine J. 2008;17(3):327-335.

37. O'Sullivan PB, Phyty GD, Twomey LT, Allison GT. Evaluation of specific stabilizing exercise in the treatment of chronic low back pain with radiologic diagnosis of spondylolysis or spondylolisthesis. Spine (Phila Pa 1976). 1997;22(24):29592967.

38. Joelson A, Hedlund R, Frennered K. Normal healthrelated quality of life and ability to work twenty-nine years after in situ arthrodesis for high-grade isthmic spondylolisthesis. $J$ Bone Joint Surg Am. 2014;96(12):e100.

39. Joelson A, Danielson BI, Hedlund R, Wretenberg P, Frennered K. Sagittal balance and health-related quality of life three decades after in situ arthrodesis for high-grade isthmic spondylolisthesis. J Bone Joint Surg Am. 2018;100(16):13571365.

40. Gollapudi PR, Kotakadira S, Nandigama PK, Karla R, Maila SK, Bugude NN. In situ posterolateral and fibular interbody fusion in high grade spondylolysthesis. $\mathrm{Br} J$ Neurosurg. 2013;27(4):454-458.

41. Noorian S, Sorensen K, Cho W. A systematic review of clinical outcomes in surgical treatment of adult isthmic spondylolisthesis. Spine J. 2018;18(8):1441-1454.

42. Wiltse LL. The paraspinal sacrospinalis-splitting approach to the lumbar spine. Clin Orthop Relat Res. 1973(91):4857.

43. Pizzutillo PD, Mirenda W, MacEwen GD. Posterolateral fusion for spondylolisthesis in adolescence. J Pediatr Orthop. 1986;6(3):311-316.

44. Johnson JR, Kirwan EO. The long-term results of fusion in situ for severe spondylolisthesis. J Bone Joint Surg Br. 1983;65(1):43-46.

45. Lee SH, Choi WG, Lim SR, Kang HY, Shin SW. Minimally invasive anterior lumbar interbody fusion followed by percutaneous pedicle screw fixation for isthmic spondylolisthesis. Spine J. 2004;4(6):644-649.

46. Ishihara H, Osada R, Kanamori M, et al. Minimum 10-year follow-up study of anterior lumbar interbody fusion for isthmic spondylolisthesis. J Spinal Disord. 2001;14(2):9199 .

47. Riouallon G, Lachaniette $\mathrm{CH}$, Poignard A, Allain J. Outcomes of anterior lumbar interbody fusion in low-grade isthmic spondylolisthesis in adults: a continuous series of 65 cases with an average follow-up of 6.6 years. Orthop Traumatol Surg Res. 2013;99(2):155-161.

48. Lassale B, Bitan F, Bex M, Deburge A. [Functional results and prognostic factors in the surgical treatment of 
degenerative lumbar stenosis]. Rev Chir Orthop Reparatrice Appar Mot. 1988;74(suppl 2):85-88.

49. Krause KL, DeDeaux C, Jung E, Than KD. Two-level reverse Bohlman transsoseous approach for treatment of symptomatic pseudarthrosis. Br J Neurosurg. 2019;33(1):8487.

50. Bohlman HH, Cook SS. One-stage decompression and posterolateral and interbody fusion for lumbosacral spondyloptosis through a posterior approach. Report of two cases. $J$ Bone Joint Surg Am. 1982;64(3):415-418.

51. Hart RA, Domes CM, Goodwin B, et al. High-grade spondylolisthesis treated using a modified Bohlman technique: results among multiple surgeons. J Neurosurg Spine. 2014;20(5):523-530.

52. Donnally CJ 3rd, Madhavan K, Butler AJ, et al. A novel technique for stabilization of high-grade spondylolisthesis with transvertebral fusion without reduction. J Clin Neurosci. 2019;60:170-175.

53. Abdu WA, Wilber RG, Emery SE. Pedicular transvertebral screw fixation of the lumbosacral spine in spondylolisthesis. A new technique for stabilization. Spine (Phila $\mathrm{Pa}$ 1976). 1994;19(6):710-715.

54. Bartolozzi P, Sandri A, Cassini M, Ricci M. One-stage posterior decompression-stabilization and trans-sacral interbody fusion after partial reduction for severe L5-S1 spondylolisthesis. Spine (Phila Pa 1976). 2003;28(11):1135-1141.

55. Grob D, Humke T, Dvorak J. Direct pediculo-body fixation in cases of spondylolisthesis with advanced intervertebral disc degeneration. Eur Spine J. 1996;5(4):281-285.

56. Minamide A, Akamaru T, Yoon ST, Tamaki T, Rhee JM, Hutton WC. Transdiscal L5-S1 screws for the fixation of isthmic spondylolisthesis: a biomechanical evaluation. J Spinal Disord Tech. 2003;16(2):144-149.

57. Zagra A, Giudici F, Minoia L, Corriero AS, Zagra L. Long-term results of pediculo-body fixation and posterolateral fusion for lumbar spondylolisthesis. Eur Spine J. 2009;18(suppl 1):151-155.

58. Logroscino CA, Tamburrelli FC, Scaramuzzo L, Schiro GR, Sessa S, Proietti L. Transdiscal L5-S1 screws for the treatment of adult spondylolisthesis. Eur Spine J. 2012;21(suppl 1):S128-S133.

59. Ferrero E, Ilharreborde B, Mas V, Vidal C, Simon AL, Mazda K. Radiological and functional outcomes of high-grade spondylolisthesis treated by intrasacral fixation, dome resection and circumferential fusion: a retrospective series of 20 consecutive cases with a minimum of 2 years follow-up. Eur Spine J. 2018;27(8):1940-1948.

60. Smith JA, Hu SS. Management of spondylolysis and spondylolisthesis in the pediatric and adolescent population. Orthop Clin North Am. 1999;30(3):487-499, ix.

61. Jiang G, Ye C, Luo J, Chen W. Which is the optimum surgical strategy for spondylolisthesis: reduction or fusion in situ? A meta-analysis from 12 comparative studies. Int J Surg. 2017;42:128-137.

62. Fan $\mathrm{G}, \mathrm{Gu} \mathrm{G}, \mathrm{Zhu} \mathrm{Y}$, et al. Minimally invasive transforaminal lumbar interbody fusion for isthmic spondylolisthesis: in situ versus reduction. World Neurosurg. 2016;90:580-587.e1.

63. Longo UG, Loppini M, Romeo G, Maffulli N, Denaro V. Evidence-based surgical management of spondylolisthesis: reduction or arthrodesis in situ. J Bone Joint Surg Am. 2014;96(1):53-58.

64. Martiniani M, Lamartina C, Specchia N. "In situ" fusion or reduction in high-grade high dysplastic developmental spondylolisthesis (HDSS). Eur Spine J. 2012;21(suppl 1):S134 S140.

65. Smith MD, Bohlman HH. Spondylolisthesis treated by a single-stage operation combining decompression with in situ posterolateral and anterior fusion. An analysis of eleven patients who had long-term follow-up. J Bone Joint Surg Am. 1990;72(3):415-421.

66. Matthiass HH, Heine J. The surgical reduction of spondylolisthesis. Clin Orthop Relat Res. 1986(203):34-44.

67. Sailhan F, Gollogly S, Roussouly P. The radiographic results and neurologic complications of instrumented reduction and fusion of high-grade spondylolisthesis without decompression of the neural elements: a retrospective review of 44 patients. Spine (Phila Pa 1976). 2006;31(2):161-169; discussion 70 .

68. Bradford DS, Boachie-Adjei O. Treatment of severe spondylolisthesis by anterior and posterior reduction and stabilization. A long-term follow-up study. J Bone Joint Surg Am. 1990;72(7):1060-1066.

69. Dick WT, Schnebel B. Severe spondylolisthesis. Reduction and internal fixation. Clin Orthop Relat Res. 1988(232):70 79.

70. Poussa M, Schlenzka D, Seitsalo S, Ylikoski M, Hurri H, Osterman K. Surgical treatment of severe isthmic spondylolisthesis in adolescents. Reduction or fusion in situ. Spine (Phila Pa 1976). 1993;18(7):894-901.

71. Petraco DM, Spivak JM, Cappadona JG, Kummer FJ, Neuwirth MG. An anatomic evaluation of L5 nerve stretch in spondylolisthesis reduction. Spine (Phila Pa 1976). 1996;21(10):1133-1138; discussion 9.

72. Schar RT, Sutter M, Mannion AF, et al. Outcome of L5 radiculopathy after reduction and instrumented transforaminal lumbar interbody fusion of high-grade L5-S1 isthmic spondylolisthesis and the role of intraoperative neurophysiological monitoring. Eur Spine J. 2017;26(3):679-690.

73. Transfeldt EE, Mehbod AA. Evidence-based medicine analysis of isthmic spondylolisthesis treatment including reduction versus fusion in situ for high-grade slips. Spine (Phila Pa 1976). 2007;32(suppl 19):S126-S129.

74. Smith JA, Deviren V, Berven S, Kleinstueck F, Bradford DS. Clinical outcome of trans-sacral interbody fusion after partial reduction for high-grade 15-s1 spondylolisthesis. Spine (Phila Pa 1976). 2001;26(20):2227-2234.

75. Boachie-Adjei O, Do T, Rawlins BA. Partial lumbosacral kyphosis reduction, decompression, and posterior lumbosacral transfixation in high-grade isthmic spondylolisthesis: clinical and radiographic results in six patients. Spine (Phila $\mathrm{Pa}$ 1976). 2002;27(6):E161-E168.

76. Gaines RW, Nichols WK. Treatment of spondyloptosis by two stage L5 vertebrectomy and reduction of L4 onto S1. Spine (Phila Pa 1976). 1985;10(7):680-686.

77. Passias PG, Poorman CE, Yang S, et al. Surgical treatment strategies for high-grade spondylolisthesis: a systematic review. Int J Spine Surg. 2015;9:50.

78. Lehmer SM, Steffee AD, Gaines RW Jr. Treatment of L5-S1 spondyloptosis by staged L5 resection with reduction and 
fusion of L4 onto S1 (Gaines procedure). Spine (Phila Pa 1976). 1994;19(17):1916-1925.

79. Obeid I, Laouissat F, Bourghli A, Boissiere L, Vital JM. One-stage posterior spinal shortening by L5 partial spondylectomy for spondyloptosis or L5-S1 high-grade spondylolisthesis management. Eur Spine J. 2016;25(2):664-670.

Corresponding Author: Kyle N. Kunze, MD, Hospital for Special Surgery, 535 East $70^{\text {th }}$ Street,
New York, NY 10021. Phone: 1 (609) 214-9245; Email:kylekunze7@gmail.com.

Published 30 June 2020

This manuscript is generously published free of charge by ISASS, the International Society for the Advancement of Spine Surgery. Copyright (C) 2020 ISASS. To see more or order reprints or permissions, see http://ijssurgery.com. 